\title{
Non-invasive sampling of water-borne hormones demonstrates individual consistency of the cortisol response to stress in laboratory zebrafish (Danio rerio)
}

\author{
H. L.E. Midttun \\ Norwegian University of Life Sciences \\ O. Overli \\ Norwegian University of Life Sciences \\ C. Tudorache \\ Leiden University \\ lan Mayer \\ Norwegian University of Life Sciences \\ I. B. Johansen ( $\Delta$ ida.johansen@nmbu.no) \\ Norwegian University of Life Sciences
}

\section{Research Article}

Keywords:

Posted Date: January 19th, 2022

DOI: https://doi.org/10.21203/rs.3.rs-1252996/v1

License: (c) (1) This work is licensed under a Creative Commons Attribution 4.0 International License.

Read Full License

Version of Record: A version of this preprint was published at Scientific Reports on April 15th, 2022. See the published version at https://doi.org/10.1038/s41598-022-10274-0. 


\section{Abstract}

Glucocorticoid (GC) stress hormones are well-known for their impact on phenotypic traits ranging from immune function to behaviour and cognition. For that reason, consistent aspects of an individual's physiological stress response (i.e. GC responsiveness) can predict major elements of life-history trajectory. Zebrafish (Danio rerio) emerge as a promising model to study such consistent trait correlations, including the development of individual stress coping styles, i.e. consistent associations between physiological and behavioral traits. However, consistency in GC responsiveness of this popular animal model remains to be confirmed. Such a study has so far been hampered by the small-bodied nature and insufficient blood volume of this species to provide repeated measurements of circulating GCs. Here, we developed a technique that allows for repeated, non-invasive sampling of individual zebrafish by quantifying GCs from holding water. Our findings indicate consistency of the magnitude of post-stress GC production over several consecutive stress events in zebrafish and that water-borne GCs accurately reflect individually consistent variation in GC responsiveness in this species.

\section{Introduction}

Throughout the vertebrate lineage, glucocorticoid "stress hormones" (GCs, mainly cortisol and corticosterone) are central in shaping major aspects of individual phenotype. Traits under GC control include immune function, reproductive investment, reallocation of energy away from somatic growth towards stress coping, and various aspects of behaviour, neural plasticity and cognition [1-13]. Numerous studies in different animal models have revealed individual consistency and a degree of heritability of the magnitude of the GC response to stress (e.g. [14-18]). In other words, individuals tend to respond to episodes of stress with the same magnitude of the GC response and this tendency is passed on to their offspring. Furthermore, in a range of animal species and taxa (e.g. teleosts, rodents, birds and pigs), physiological and behavioral responses to stress are associated in such a manner that distinct stress coping styles along a reactive-proactive continuum can be identified [19-24]. Various terms are used to categorize individuals employing consistently different reaction norms in response to changes in the environment, but when physiological correlates (e.g. GC responsiveness) of consistent behavioral patterns are concerned, animals are commonly classified as either "reactive" or "proactive" based on their distribution along a shy-bold continuum (for reviews, see [19, 24-26]). Stress coping styles are thus defined as a set of behavioral and physiological responses to stress that are consistently employed by one individual across unrelated and temporally separated situations [25]. Reactive individuals are characterized by having a high post-stress GC production whereas proactive individuals respond to stress with low GC production. Ultimate and proximate mechanisms underlying such consistent trait correlations are probably complex and remain largely unknown. To understand these intricate mechanisms, we need novel model systems allowing the use of advanced molecular-genetic tools.

Among the vertebrate lineage, teleost fish (e.g. salmonids, zebrafish; Danio rerio, medaka; Oryzias latipes, goldfish; Carassius auratus) are now rapidly complementing or replacing rodent models in scientific 
disciplines like neurobiology, toxicology and immunology. In particular, the zebrafish is becoming an increasingly popular laboratory animal model in neurobiological and behavioural studies [27]. Considering the rapid development of behavioral screening [28-30] and molecular genetic tools [31] available for this species, zebrafish emerge as a promising model to reveal mechanisms underlying consistent phenotypic trait correlations. Several studies have demonstrated a strong covariation between behaviour and post-stress cortisol levels in zebrafish [22, 32]. Moreover, distinct behavioral patterns appear to be generally consistent across contexts and time and influenced by selective pressures in zebrafish [33-35].

In view of the above, covariation in behaviour and GCs and consistency in behavior are indicative of stress coping styles in zebrafish. However, to comply with the strict definition of stress coping style [25], it remains to be determined whether physiological stress responsiveness (i.e. GC responsiveness) is a consistent trait in this species. Such a study has been hampered by the fact that zebrafish are smallbodied animals with blood volumes insufficient to provide repeated measurements of circulating GCs. Instead, whole-body GC levels have been used for several decades to assess the stress response of zebrafish [36-38], like in other small-bodied fish such as young salmonids [39] and flatfish [40]. These GC measurements are however terminal, without the possibility of repetition over time. This constraint has thus entailed a significant knowledge gap regarding individual consistency of the stress response, since techniques that allow for repeated, non-invasive sampling from in the same individuals across conditions are essential in this context [41].

For teleost fish, a solution may be provided by extracting GCs from the fish holding water. In aquatic organisms, circulating steroid hormones are continuously released by the organism into the surrounding water. For example, Félix et al (2013) validated that GC levels in the holding water reliably reflect baseline (i.e. resting levels) circulating plasma hormone levels in zebrafish. Whether GC levels in holding water accurately reflects post stress GC production has however not previously been studied in such smallbodied fish as $D$. rerio. If it does, non-invasive repeated measures of GC responsiveness can be done from the same individual.

By repeatedly measuring post stress cortisol levels (the dominating GC in zebrafish) non-invasively, we show that GC responsiveness is a consistent trait in zebrafish. In addition, water cortisol levels closely correlated to whole-body cortisol measured terminally, suggesting that GC levels in holding water accurately reflects post stress GC production. The consistency of GC responsiveness in this animal model allows for future generation of zebrafish stains differing in stress reactivity that can serve as valuable models for studying the biology underlying stress coping styles, including individual vulnerability to stress-related diseases.

\section{Materials And Methods}

Ethics statement 
Zebrafish were maintained and handled according to the guidelines from the Zebrafish Information Network (ZFIN, http://zfin.org). All experimental protocols were approved by the Norwegian Animal Research Authority (NARA), following the Norwegian laws and regulations controlling experiments and procedures on live animals in Norway (permit number 11241). The study was carried out in compliance with the ARRIVE guidelines.

\section{Animals and housing}

A total of 25 female and 24 male adult AB wild-type zebrafish, obtained from the Department of Production Animal Clinical Sciences, the Norwegian University of Life Sciences (Oslo, Norway), were housed in a recirculating Z Mod system (Marine Biotech), at a 14:10 light:dark cycle. Water temperature was kept at $28^{\circ} \mathrm{C}, \mathrm{pH}$ and conductivity were maintained at 7.4-7.6 and $500 \mu \mathrm{S}$ respectively as according to the Zebrafish International Resource Center guidelines [43]. Fish were fed twice daily with commercial pelleted food (Special Diets Service, Witham, Great Britain) and twice with Artemia nauplii (Sep-art, Ocean Nutrition, Belgium). Prior to experiments, fish were starved for at least 12 hours. Females and males were kept in separate tanks at a recommended density of 5 fish/L [44].

\section{VIE-Tagging}

All fish were anaesthetized in $0.02 \%$ tricaine methanesulfonate (MS-222; Sigma, St. Louis Missouri, USA). Fish were subsequently injected subcutaneously with Fluorescing Visible Implant Elastomer (VIE) tags (Northwest Marine Technology Inc., Shaw Island Washington, USA) following the method by [45], either adjacent to the dorsal fin, at the abdomen, or both, creating individually identifiable patterns. Immediately after recovery from surgery, fish were transferred to a holding tank containing clean system water. Testing was commenced after a minimum of 10 days recovery period. All fish were regularly visually examined for infections and open wounds during the recovery period. All individuals had healed completely before the start of testing.

\section{Stress testing and collection of holding water for cortisol analysis}

Fish were subjected to four consecutive stress tests in total separated by a minimum of 6 days, and between 08:00 and 11:00 to control for diel fluctuations in cortisol. The experimental and analytical protocols are illustrated in Figure 1, with details following below. Initially, fish were individually netted from the holding tanks and transferred to a $250 \mathrm{~mL}$ glass beaker filled with $50 \mathrm{~mL}$ of system water containing a stirring magnet and placed on a magnetic stirrer. A UV flashlight (Northwest Marine Technology Inc., Shaw Island Washington, USA) was used to identify the fish before the stress test was initiated: the stirrer (VWR, Radnor Pennsylvania, USA) was set at the lowest stirring speed (100 RPM) and kept at this speed for 20 minutes. This speed was sufficient to induce constant swimming behaviour, interrupted by periods of burst-and-coast behaviour. After the forced swimming period, fish were netted to a $100 \mathrm{~mL}$ plastic beaker (VWR, Radnor Pennsylvania, USA) containing $50 \mathrm{~mL}$ of system water. The beaker was connected to a flow-in and flow-out tube, with the flow-in tube connected to system water through a 
valve. This flow-through system was set at a slow, constant flow of $0.3 \mathrm{~L} / \mathrm{min}$ during the first 15 minutes after the stress test was finalized. The flow-through was then shut off and the fish was kept in the beaker for 1 hour to allow cortisol to exit and accumulate into the water. Fish were then transferred back to their holding tanks until subsequent stress tests. The water was then collected in $50 \mathrm{~mL}$ Falcon tubes (Corning, Corning New York, USA) and kept at -20凶C for a later analysis of cortisol concentration. Following the 4th consecutive stress test, fish were immediately euthanized in an overdose of tricaine methanesulfonate ( $1 \mathrm{~g} / \mathrm{L}$, Sigma, St. Louis Missouri, USA). Fish were then weighed, and fork length was measured before they were frozen on dry ice. All fish were kept at -80囚C until further analysis of whole-body cortisol.

\section{Cortisol extraction}

Cortisol was extracted from water using C18 solid-phase extraction cartridges (Waters, Milford Massachusetts, USA). Cartridges were activated with $5 \mathrm{~mL}$ methanol and rinsed with $10 \mathrm{~mL} \mathrm{MQ}$ water. Water samples were thawed at room temperature before being pumped through the cartridges by a peristaltic pump at $10 \mathrm{ml} / \mathrm{min}$ for $5 \mathrm{~min}$ (SCl-Q 323, Watson-Marlow, Falmouth, Great Britain). Cartridges were then rinsed with $10 \mathrm{~mL} M Q$ water using a syringe and all excess water was expelled by pressing air through the cartridge, before storing the cartridge at $-20 \llbracket \mathrm{C}$ until further use.

Cartridges were thawed at room temperature before elution with $10 \mathrm{~mL}$ methanol into glass test tubes (130 x 16 mm, VWR, Radnor Pennsylvania, USA). Methanol was evaporated in an evaporator (ReactiTherm III heater, Thermo Scientific, Waltham Massachusetts, USA) at 25هC in a fume hood. Steroid hormones were reconstituted in $500 \mu \mathrm{L}$ assay buffer supplied in the ELISA kit (DetectX®, Arbor Assays, Ann Arbor Michigan, USA). Test tubes were vortexed for 30 seconds, left on ice for 30 minutes, vortexed again and samples were transferred to microcentrifuge tubes (Eppendorf, Hamburg, Germany). All samples were stored at $-20 \unrhd \mathrm{C}$ until further analysis.

Cortisol extraction from whole-body was carried out according to Ramsay et al. (2006) with few modifications. Nine male and eight female fish were used for the extraction. Briefly, fish were thawed and transferred to a $5 \mathrm{~mL}$ microtube (Eppendorf, Hamburg, Germany) with $750 \mu \mathrm{L} \mathrm{MQ}$ water, the fish were homogenized using a handheld homogenizer (VDI 12, VWR, Radnor Pennsylvania, USA) for 90-120 seconds. The homogenizer was cleaned with $\mathrm{MQ}$ and ethanol between samples. After homogenization 4 $\mathrm{mL}$ of diethyl ether was added, before the samples were vortexed vigorously and poured into glass test tubes. An additional $4 \mathrm{~mL}$ of diethyl ether was added to the test tubes before they were vortexed for 30 seconds and put on ice. The samples were subsequently centrifuged for 2 minutes at $1320 \mathrm{~g}$ and stored at $-80 \otimes \mathrm{C}$ for 10 minutes to freeze the tissue so that supernatant containing extracted cortisol could be transferred to a new test tube. Following freezing, the diethyl ether was evaporated in an evaporator (Reacti-Therm III heater, Thermo Scientific, Waltham Massachusetts, USA) at 25खC in a fume hood and extracted steroid hormones were reconstituted in $1 \mathrm{~mL}$ of assay buffer supplied in the ELISA kit (DetectX®, Arbor Assays). All samples were stored at -20®C until further use.

\section{ELISA}


Whole-body and water cortisol extractions were assayed using a commercial ELISA kit (Arbor Assays, Ann Arbor Michigan, USA). Whole-body cortisol extractions were diluted 1:25 while water cortisol extractions were diluted 1:5 both with assay buffer supplied in the ELISA kit. The ELISA was run according to manufacturer's protocol, and all samples were run in duplicates.

\section{Statistical analysis}

All data were tested for normality by a Shapiro-Wilk test. Consistency in cortisol responsiveness between consecutive stress tests was tested by linear regression. Correlation was determined by a Pearson $r$ test in case of a normal distribution and by a Spearman rank test in case of a non-normal distribution.

Differences in cortisol response and body weights between females and males were tested by a twotailed unpaired t-test, with Welch's correction for unequal standard deviations when appropriate. Significance was accepted at $p<0.05$. All values are given as mean \pm SEM. All statistical analyses were performed in GraphPad Prism 7.0. (GraphPad Software, San Diego, CA, USA).

\section{Results}

Cortisol-response to stress is variable between, but consistent within individual zebrafish

For all fish across all tests performed in this study, post stress whole-body cortisol levels ranged between 14.9 and $100.6 \mathrm{ng} \mathrm{g}^{-1}$ fish, with an average of $44.9 \mathrm{ng} \mathrm{g}^{-1}$. That is similar to or higher than post stress whole-body cortisol levels previously reported for this species $[46,47]$. Cortisol levels were also markedly higher than resting whole-body cortisol levels $\left(4-5 \mathrm{ng} \mathrm{g}^{-1}\right)$ reported for zebrafish $[46,47]$.

To test for consistency in post-stress cortisol production, holding water from individual zebrafish was collected three times following three separate stress tests on the same individuals. Moreover, a fourth stress test was conducted to investigate consistency between water cortisol and whole-body cortisol measurements.

There were strong positive correlations in the measured cortisol levels between the separate tests for both male and female zebrafish (Fig. 2). More precisely, cortisol levels in the holding water from stress test 1 was a predictor of water cortisol levels in stress tests 2 (males: $R^{2}=0.56, p<0.001$, Fig. $2 A$ and females: $R^{2}=0.41, p<0.01$, Fig. $2 B$ ) and 3 (males: $R^{2}=0.41, p<0.01$, Fig. $2 C$ and females: $R^{2}=0.31, p<0.05$, Fig. $2 D$ ). Average water cortisol levels from the three initial stress tests significantly predicted whole-body cortisol levels in males $\left(R^{2}=0.52, p<0.05\right.$, Fig. $\left.2 E\right)$ but this association did not quite reach statistical significance in females $\left(R^{2}=0.40, p=0.09\right.$, Fig. $\left.2 F\right)$.

Cortisol levels and between-individual variation is higher in males compared to females

Cortisol levels in both water (un-paired t-test: welch-corrected $t=3.57, d f=8.62, p<0.001$, Fig.3A) and wholebody (un-paired t-test: welch-corrected t=2.38, df=8.82, $p<0.05$, Fig.3B) were markedly lower for females compared to males. Moreover, individual variation in post-stress cortisol levels measured in holding water 
$(F=22.87, D F n=8, D f d=7, p<001)$ and whole-body $(F=21.93, D F n=8, D f d=7, p<001)$ was considerably higher in males compared to females (Fig.3). There was no significant difference between male and female bodyweight (mean \pm SEM for males: $0.428 \pm 0.04395 \mathrm{~g}$, females: $0.6083 \pm 0.05491 \mathrm{~g}, \mathrm{p}=0.1071$ ) for the fish tested in this study (data not shown).

\section{Discussion}

In this study we observed that GC responsiveness to stress is a consistent individual trait in zebrafish. This new finding was determined using a technique that facilitated repeated, non-invasive sampling of the same individuals, followed by the extraction and quantification of GCs from the holding water of isolated stressed fish. We also confirmed a previously reported strong sexual dimorphism with regards to individual response patterns, (sexual dimorphism), with female zebrafish responding to stress with lower levels of cortisol compared to male fish [22, 48,49]. Our findings indicate consistency of the magnitude of post-stress GC production over several successive stress events in zebrafish, when surveyed under standardized conditions. The methods customized in this study validate that post-stress GC production and release can be reliably reflected by water-borne hormone levels. Furthermore, individual GC profiles remain consistent over several weeks, until study termination allowing for final confirmation by wholebody hormone levels.

A standardized sampling regime together with a sensitive and precise analytical method were key to the verification of our findings. Our data expand on two recent reviews and meta-analyses identifying considerable variation between studies regarding consistency of GC secretion $[41,50]$. Both metaanalyses found that GC repeatability measures vary between different taxa, with the highest repeatability found in amphibians. However, both reviews observe that an absolute majority of published studies on within-individual consistency of GC titers are conducted in birds ( $75-80 \%$ of studies), followed by mammals $(15 \%)$ and poikilotherms $(<10 \%)$. The higher consistency was generally found in stress-induced versus resting GC levels, while pronounced differences in the magnitude of the physiological response to stress is a consistent observation between species. These and other studies suggest that there are fundamental evolutionary pressures which promote a diversity of persistent stress coping styles and response strategies among vertebrates $[16,24,25,51]$. The relative scarcity of studies in fish, amphibians, and reptiles highlights the need for further research in comparative models to clarify the evolutionary origin of this trend.

Indeed, in several teleost species, GC responsiveness to stress has been reported to correlate with other physiological and behavioral responses to stress [24, 26, 52]. For example, GC responsiveness is a highly consistent trait in rainbow trout (Oncorhynchus mykiss) and post stress GC levels accurately predict behavioral correlates of personality [23, 53, 54], brain function, and neural plasticity [55-57]. For instance, GC responsiveness is generally a reliable predictor of social position, with low responding individuals usually becoming socially dominant (Pottinger and Carrick, 2001), but see Ruiz-Gomez et al. [2004] for an example of context-dependency in this pattern. 
Previous studies have correlated singularly measured post-stress GC levels with behavioral indicators of stress coping style (as defined by Koolhaas et al. 1999) in zebrafish. For example, Tudorache et al. [32] showed that proactive zebrafish larvae respond to stress with lower whole-body cortisol levels and a faster recovery back to baseline levels than reactive individuals. Similarly, Wong et al. [46] found that post-stress GC responsiveness was lower in zebrafish selected to display low (LSB, presumably proactive) compared to high (HSB, presumably reactive) stationary behaviour. Although behavioral variation was not assessed in the current study, these previous observations along with the individual consistency in GC responsiveness reported here is consistent with the existence of stress coping styles in zebrafish. It is important to note that the fish tested in the current study came from an inbred lab strain of zebrafish and that the genetic variation in this lab population is likely to be considerably lower than in wild zebrafish. It is a reasonable assumption that GC responsiveness, being at least in part genetically based [58-60] is likely to be even more variable in undomesticated fish populations.

Glucocorticoid hormones are major neuroendocrine integrators influencing vital biological processes including reproduction, growth, metabolism, immune function, cognition and emotion $[2,61-63]$. Hence, individual consistency in magnitude of the GC response to stress (as well as the possibility to accurately measure it) in zebrafish has several research implications. Firstly, in a fundamental perspective zebrafish emerge as a promising model to reveal the proximate and ultimate mechanisms underlying consistent phenotypic trait correlations. Secondly, zebrafish is already a favored animal model for high throughput research in many fields of biology, including stress-related diseases such as anxiety, depression, and cardiovascular disease [64-67]. Also with respect to infection biology, given the immune-modulating and neurodegenerative nature of GCs, stress reactivity is considered a likely predictor of disease vulnerability and trajectory $[68,69]$. Scientific questions often relate to how a particular pathogen or environmental condition affects disease vulnerability and progression. In many cases, any individual variation in severity of disease is regarded as noise and fail to appreciate such variation as a possible source of important biological information and knowledge. In this context, if correctly implemented in experimental design, non-invasive measurements of GC responsiveness may reveal whether individually different outcomes reflect inherent biological variation or unintended treatment variation.

In summary, the current study shows that water-borne GCs accurately reflect individually consistent variation in GC responsiveness in zebrafish. This non-invasive approach has several advantages, both for research and animal welfare (reduction of animal numbers). Further, adopting this non-invasive method will result in the intact fish (and tissues) being available for subsequent behavioral, morphometric, physiological, or molecular analysis. Thus, zebrafish can provide unique insights into not only the molecular-genetic mechanisms underlying phenotypic variation, but also how such variation is constrained by selection [70]. Further validation of this method is provided by the observed sex differences with higher post-stress cortisol levels in male compared to female zebrafish [22, 48, 49]. The methods described here are also applicable to a wide range of other small-bodied aquatic vertebrates, a category which provides almost inexhaustible diversity for the study of stress responses as adaptive traits enabling the organism to cope with a changing environment. 


\section{Declarations}

Acknowledgements

We would like to thank Ana Carolina Sulen Tavara and Arturas Kavaliauskis for valuable help with zebrafish husbandry.

\section{Author contributions}

I.B.J. and O.O. conceived the project. H.E.M., I.B.J., I.M., C.T. and O.O. contributed to the design of the experiments. H.E.M., I.B.J. and I.M. performed the experiments and contributed to the collection and analysis of data. H.E.M. and I.B.J conducted statistical analysis. O.O., I.B.J. H.E.M. and C.T. wrote the paper. All authors edited the paper.

\section{Additional information}

The authors declare no competing interests. Funding provided by The Research Council of Norway and Norwegian University of Life Sciences, grant number 250048

\section{Data availability}

The datasets generated during and analyzed during the current study are available in the NMBU Open Research Data repository, [https://dataverse.no/dataset.xhtml?persistentld=doi:10.18710/HM1HXV].

\section{References}

1. Pickering A, Pottinger TG. Stress responses and disease resistance in salmonid fish: effects of chronic elevation of plasma cortisol. Fish physiology and biochemistry. 1989;7(1):253-8.

2. Sapolsky RM, Romero LM, Munck AU. How do glucocorticoids influence stress responses? Integrating permissive, suppressive, stimulatory, and preparative actions. Endocrine reviews. 2000;21(1):55-89.

3. Korte S. Corticosteroids in relation to fear, anxiety and psychopathology. Neuroscience \& Biobehavioral Reviews. 2001;25(2):117-42.

4. Øverli $\varnothing$, Kotzian S, Winberg S. Effects of cortisol on aggression and locomotor activity in rainbow trout. Hormones and Behavior. 2002;42(1):53-61.

5. De Kloet ER, Joëls M, Holsboer F. Stress and the brain: from adaptation to disease. Nature reviews neuroscience. 2005;6(6):463-75.

6. Het S, Ramlow G, Wolf OT. A meta-analytic review of the effects of acute cortisol administration on human memory. Psychoneuroendocrinology. 2005;30(8):771-84.

7. Summers $\mathrm{CH}$, Watt MJ, Ling TL, Forster GL, Carpenter RE, Korzan WJ, et al. Glucocorticoid interaction with aggression in non-mammalian vertebrates: reciprocal action. European journal of pharmacology. 2005;526(1-3):21-35. 
8. Landys MM, Ramenofsky M, Wingfield JC. Actions of glucocorticoids at a seasonal baseline as compared to stress-related levels in the regulation of periodic life processes. General and comparative endocrinology 2006.;148(2):132-49.

9. Lupien SJ, Maheu F, Tu M, Fiocco A, Schramek TE. The effects of stress and stress hormones on human cognition: Implications for the field of brain and cognition. Brain and Cognition. 2007;65(3):209-37.

10. Crossin GT, Phillips RA, Lattin CR, Romero LM, Williams TD. Corticosterone mediated costs of reproduction link current to future breeding. General comparative endocrinology 2013.;193:112-20.

11. Gorissen M, Flik G. The endocrinology of the stress response in fish: an adaptation-physiological view. In: Schreck CB, Tort L, Farrell AP, Brauner CJ, editors. Fish physiology. 35: Elsevier; 2016. p. 75111.

12. Cain DW, Cidlowski JA. Immune regulation by glucocorticoids. Nature Reviews Immunology. 2017;17(4):233-47.

13. Campbell JH, Dixon B, Whitehouse LM. The intersection of stress, sex and immunity in fishes. Immunogenetics. 2021:1-19.

14. Satterlee $D$, Johnson W. Selection of Japanese quail for contrasting blood corticosterone response to immobilization. Poultry Science. 1988;67(1):25-32.

15. Pottinger T, Carrick T. Modification of the plasma cortisol response to stress in rainbow trout by selective breeding. General and Comparative Endocrinology. 1999;116(1):122-32.

16. Cockrem JF. Individual variation in glucocorticoid stress responses in animals. General Comparative Endocrinology. 2013;181:45-58.

17. Narayan EJ, Cockrem JF, Hero J-M. Repeatability of baseline corticosterone and short-term corticosterone stress responses, and their correlation with testosterone and body condition in a terrestrial breeding anuran (Platymantis vitiana). Comparative Biochemistry Physiology Part A: Molecular Integrative Physiology. 2013;165(2):304-12.

18. Stedman J, Hallinger K, Winkler D, Vitousek M. Heritable variation in circulating glucocorticoids and endocrine flexibility in a free-living songbird. Journal of evolutionary biology. 2017;30(9):1724-35.

19. Koolhaas JM, Korte SM, De Boer SF, Van Der Vegt BJ, Van Reenen CG, Hopster H, et al. Coping styles in animals: current status in behavior and stress-physiology. Neurosci Biobehav Rev. 1999;23(7):925-35. Epub 1999/12/02. PubMed PMID: 10580307.

20. Cockrem JF. Stress, corticosterone responses and avian personalities. Journal of Ornithology. 2007;148(2):169-78.

21. Castanheira MF, Herrera M, Costas B, Conceição LE, Martins Cl. Linking cortisol responsiveness and aggressive behaviour in gilthead seabream Sparus aurata: Indication of divergent coping styles. Applied Animal Behaviour Science. 2013;143(1):75-81.

22. Wong RY, French J, Russ JB. Differences in stress reactivity between zebrafish with alternative stress coping styles. Royal Society open science. 2019;6(5):181797. 
23. Øverli $\varnothing$, Korzan WJ, Höglund E, Winberg S, Bollig H, Watt M, et al. Stress coping style predicts aggression and social dominance in rainbow trout. Hormones and Behavior. 2004;45(4):235-41.

24. Øverli $\emptyset$, Sørensen C, Pulman KG, Pottinger TG, Korzan W, Summers CH, et al. Evolutionary background for stress-coping styles: relationships between physiological, behavioral, and cognitive traits in non-mammalian vertebrates. Neuroscience Biobehavioral Reviews. 2007;31(3):396-412.

25. Koolhaas J, De Boer S, Coppens C, Buwalda B. Neuroendocrinology of coping styles: towards understanding the biology of individual variation. Frontiers in neuroendocrinology. 2010;31(3):30721.

26. Castanheira MF, Conceição LE, Millot S, Rey S, Bégout ML, DamsgAard B, et al. Coping styles in farmed fish: consequences for aquaculture. Reviews in Aquaculture. 2017;9(1):23-41.

27. Egan RJ, Bergner CL, Hart PC, Cachat JM, Canavello PR, Elegante MF, et al. Understanding behavioral and physiological phenotypes of stress and anxiety in zebrafish. Behavioural brain research. 2009;205(1):38-44.

28. Midttun HL, Vindas MA, Nadler LE, Øverli Ø, Johansen IB. Behavioural effects of the common braininfecting parasite Pseudoloma neurophilia in laboratory zebrafish (Danio rerio). Scientific reports. 2020;10(1):1-9.

29. Orger MB, Gahtan E, Muto A, Page-McCaw P, Smear MC, Baier H. Behavioral screening assays in zebrafish. Methods in cell biology. 2004;77:53-68.

30. Cachat J, Stewart A, Grossman L, Gaikwad S, Kadri F, Chung KM, et al. Measuring behavioral and endocrine responses to novelty stress in adult zebrafish. Nature protocols. 2010;5(11):1786-99.

31. Sassen WA, Köster RW, Genetics. A molecular toolbox for genetic manipulation of zebrafish. Advances in Genomics. 2015;5:151.

32. Tudorache C, ter Braake A, Tromp M, Slabbekoorn H, Schaaf MJ. Behavioral and physiological indicators of stress coping styles in larval zebrafish. Stress. 2015;18(1):121-8.

33. Roy T, Shukla R, Bhat A. Risk-taking during feeding: between-and within-population variation and repeatability across contexts among wild zebrafish. Zebrafish. 2017;14(5):393-403.

34. Roy T, Bhat A. Population, sex and body size: determinants of behavioural variations and behavioural correlations among wild zebrafish Danio rerio. Royal Society open science. 2018;5(1):170978.

35. Conradsen C, Walker JA, Perna C, McGuigan K. Repeatability of locomotor performance and morphology-locomotor performance relationships. Journal of Experimental Biology. 2016;219(18):2888-97.

36. Pottinger T, Calder G. Physiological stress in fish during toxicological procedures: a potentially confounding factor. Environmental Toxicology and Water Quality. 1995;10(2):135-46.

37. Ramsay JM, Feist GW, Varga ZM, Westerfield M, Kent ML, Schreck CB. Whole-body cortisol is an indicator of crowding stress in adult zebrafish, Danio rerio. Aquaculture. 2006;258(1-4):565-74.

38. von Krogh K, Sørensen C, Nilsson GE, Øverli Ø. Forebrain cell proliferation, behavior, and physiology of zebrafish, Danio rerio, kept in enriched or barren environments. Physiology and behavior. 
2010;101(1):32-9.

39. de Jesus EGT, Hirano T. Changes in whole body concentrations of cortisol, thyroid hormones, and sex steroids during early development of the chum salmon, Oncorhynchus keta. General and comparative endocrinology. 1992;85(1):55-61.

40. de Jesus EG, Hirano T, Inui Y. Changes in cortisol and thyroid hormone concentrations during early development and metamorphosis in the Japanese flounder, Paralichthys olivaceus. General and comparative endocrinology. 1991;82(3):369-76.

41. Taff CC, Schoenle LA, Vitousek MN. The repeatability of glucocorticoids: a review and meta-analysis. General and comparative endocrinology. 2018;260:136-45.

42. Félix AS, Faustino Al, Cabral EM, Oliveira RF. Noninvasive measurement of steroid hormones in zebrafish holding-water. Zebrafish. 2013;10(1):110-5.

43. Westerfield M, Book T. A guide for the laboratory use of zebrafish Danio (Brachydanio) rerio: Eugene (OR): University of Oregon Press; 1995.

44. Pavlidis M, Digka N, Theodoridi A, Campo A, Barsakis K, Skouradakis G, et al. Husbandry of zebrafish, Danio rerio, and the cortisol stress response. Zebrafish. 2013;10(4):524-31.

45. Hohn C, Petrie-Hanson L. Evaluation of visible implant elastomer tags in zebrafish (Danio rerio). Biology Open. 2013;2(12):1397-401.

46. Wong RY, French J, Russ JB. Differences in stress reactivity between zebrafish with alternative stress coping styles. Royal Society open science. 2019;6(5):181797-. doi: 10.1098/rsos.181797. PubMed PMID: 31218026.

47. Pavlidis M, Theodoridi A, Tsalafouta A. Neuroendocrine regulation of the stress response in adult zebrafish, Danio rerio. Prog Neuropsychopharmacol Biol Psychiatry. 2015;60:121-31. Epub 2015/03/10. doi: 10.1016/j.pnpbp.2015.02.014. PubMed PMID: 25748166.

48. Rambo CL, Mocelin R, Marcon M, Villanova D, Koakoski G, de Abreu MS, et al. Gender differences in aggression and cortisol levels in zebrafish subjected to unpredictable chronic stress. Physiology and Behavior. 2017;171:50-4.

49. Oswald ME, Drew RE, Racine M, Murdoch GK, Robison BD. Is behavioral variation along the bold-shy continuum associated with variation in the stress axis in zebrafish? Physiological Biochemical Zoology. 2012;85(6):718-28.

50. Schoenemann KL, Bonier F. Repeatability of glucocorticoid hormones in vertebrates: a meta-analysis. PeerJ. 2018;6:e4398.

51. Taff CC, Vitousek MN. Endocrine flexibility: optimizing phenotypes in a dynamic world? Trends in ecology and evolution. 2016;31(6):476-88.

52. Samaras A, Dimitroglou A, Sarropoulou E, Papaharisis L, Kottaras L, Pavlidis M. Repeatability of cortisol stress response in the European sea bass (Dicentrarchus labrax) and transcription differences between individuals with divergent responses. Scientific reports. 2016;6(1):1-11. 
53. Øverli $\varnothing$, Pottinger TG, Carrick TR, Øverli E, Winberg S. Differences in behaviour between rainbow trout selected for high-and low-stress responsiveness. Journal of Experimental Biology. 2002;205(3):391-5.

54. Moreira P, Pulman KG, Pottinger TG. Extinction of a conditioned response in rainbow trout selected for high or low responsiveness to stress. Hormones and Behavior. 2004;46(4):450-7.

55. Øverli Ø, Pottinger TG, Carrick TR, Øverli E, Winberg S. Brain monoaminergic activity in rainbow trout selected for high and low stress responsiveness. Brain, behavior and evolution. 2001;57(4):214-24.

56. Schjolden J, Winberg S, evolution. Genetically determined variation in stress responsiveness in rainbow trout: behavior and neurobiology. Brain, behavior and evolution. 2007;70(4):227-38.

57. Johansen IB, Sørensen C, Sandvik GK, Nilsson GE, Höglund E, Bakken M, et al. Neural plasticity is affected by stress and heritable variation in stress coping style. Comparative Biochemistry Physiology Part D: Genomics Proteomics. 2012;7(2):161-71.

58. Tanck MW, Vermeulen K-J, Bovenhuis $\mathrm{H}$, Komen $\mathrm{H}$. Heredity of stress-related cortisol response in androgenetic common carp (Cyprinus carpio L.). Aquaculture. 2001;199(3-4):283-94.

59. Fevolden SE, Refstie T, Røed KH. Selection for high and low cortisol stress response in Atlantic salmon (Salmo salar) and rainbow trout (Oncorhynchus mykiss). Aquaculture. 1991;95(1-2):53-65.

60. Khan UW, Øverli Ø, Hinkle PM, Pasha FA, Johansen IB, Berget I, et al. A novel role for pigment genes in the stress response in rainbow trout (Oncorhynchus mykiss). Scientific reports. 2016;6(1):1-11.

61. Whirledge S, Cidlowski JA. Glucocorticoids and reproduction: traffic control on the road to reproduction. Trends in Endocrinology Metabolism. 2017;28(6):399-415.

62. Ortega VA, Mercer EM, Giesbrecht GF, Arrieta M-C. Evolutionary significance of the neuroendocrine stress axis on vertebrate immunity and the influence of the microbiome on early-life stress regulation and health outcomes. Frontiers in Microbiology. 2021;12:768.

63. McEwen BS, Biron CA, Brunson KW, Bulloch K, Chambers WH, Dhabhar FS, et al. The role of adrenocorticoids as modulators of immune function in health and disease: neural, endocrine and immune interactions. Brain Research Reviews. 1997;23(1-2):79-133.

64. Maximino C, de Brito TM, da Silva Batista AW, Herculano AM, Morato S, Gouveia Jr A. Measuring anxiety in zebrafish: a critical review. Behavioural brain research. 2010;214(2):157-71.

65. Lu S, Hu M, Wang Z, Liu H, Kou Y, Lyu Z, et al. Generation and application of the zebrafish heg1 mutant as a cardiovascular disease model. Biomolecules. 2020;10(11):1542.

66. Langenbacher AD, Shimizu H, Hsu W, Zhao Y, Borges A, Koehler C, et al. Mitochondrial calcium uniporter deficiency in zebrafish causes cardiomyopathy with arrhythmia. Frontiers in Physiology. 2020;11.

67. Fontana BD, Mezzomo NJ, Kalueff AV, Rosemberg DB. The developing utility of zebrafish models of neurological and neuropsychiatric disorders: A critical review. Experimental Neurology. 2018;299:157-71. doi: https://doi.org/10.1016/j.expneurol.2017.10.004. 
68. de Boer SF, Buwalda B, Koolhaas JM. Untangling the neurobiology of coping styles in rodents:

Towards neural mechanisms underlying individual differences in disease susceptibility. Neurosci

Biobehav Rev. 2017;74(Pt B):401-22. Epub 2016/07/13. doi: 10.1016/j.neubiorev.2016.07.008.

PubMed PMID: 27402554.

69. Koolhaas JM. Coping style and immunity in animals: making sense of individual variation. Brain Behav Immun. 2008;22(5):662-7. Epub 2008/04/09. doi: 10.1016/j.bbi.2007.11.006. PubMed PMID: 18395410.

70. Baker MR, Goodman AC, Santo JB, Wong RY. Repeatability and reliability of exploratory behavior in proactive and reactive zebrafish, Danio rerio. Scientific reports. 2018;8(1):1-9.

\section{Figures}

(1)

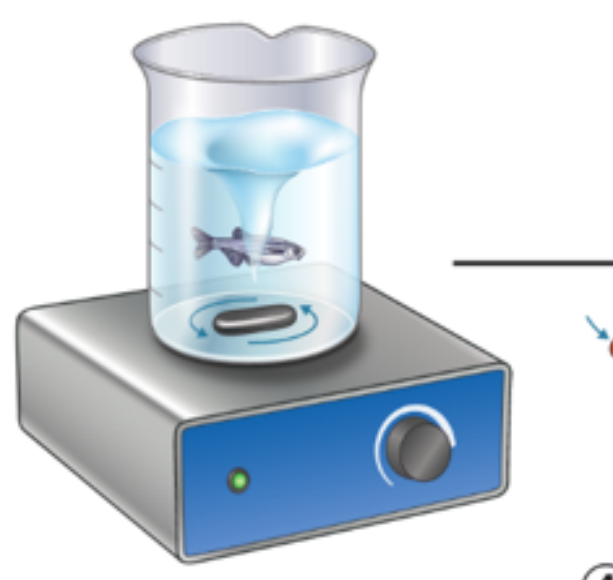

(4)
(2)

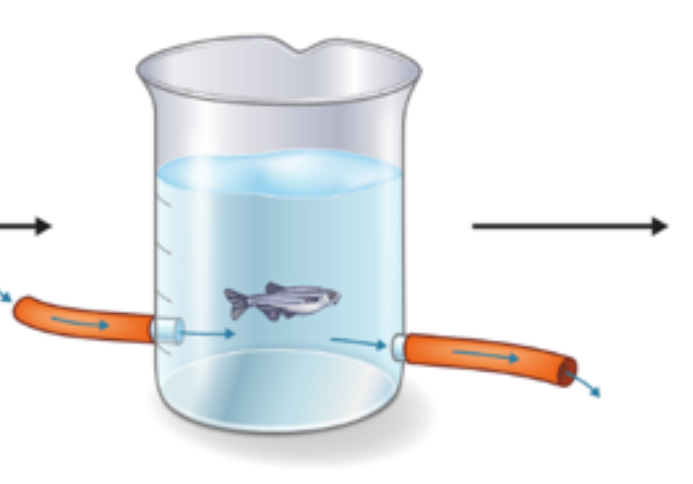

(5)

Hydrophobic analytes

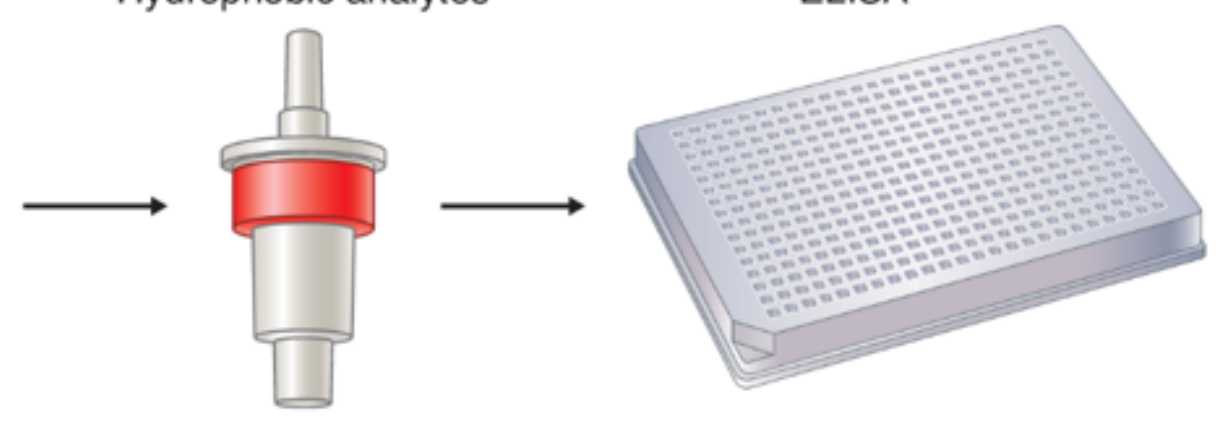

(3)

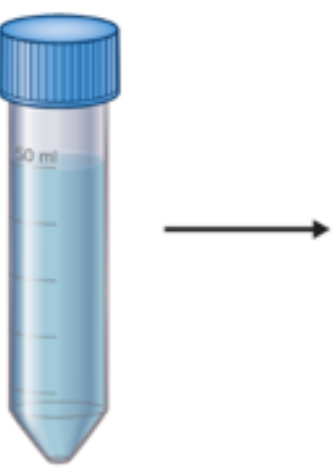

\section{Figure 1}

Experimental and analytical protocol for analysis of water-borne cortisol. 1) Fish were stressed in a glass beaker containing a stirring magnet and placed on a magnetic stirrer running at 100 RPM 2) before transferred to a plastic beaker connected to a flow-in and flow-out tube where water flow was shut off to allow cortisol to exit into the water. 3) Outflow water was then collected in $50 \mathrm{~mL}$ Falcon tubes 4) and cortisol extracted using C18 solid-phase extraction cartridges. 5) Water cortisol extractions were assayed using a commercial ELISA kit. 
A

Males
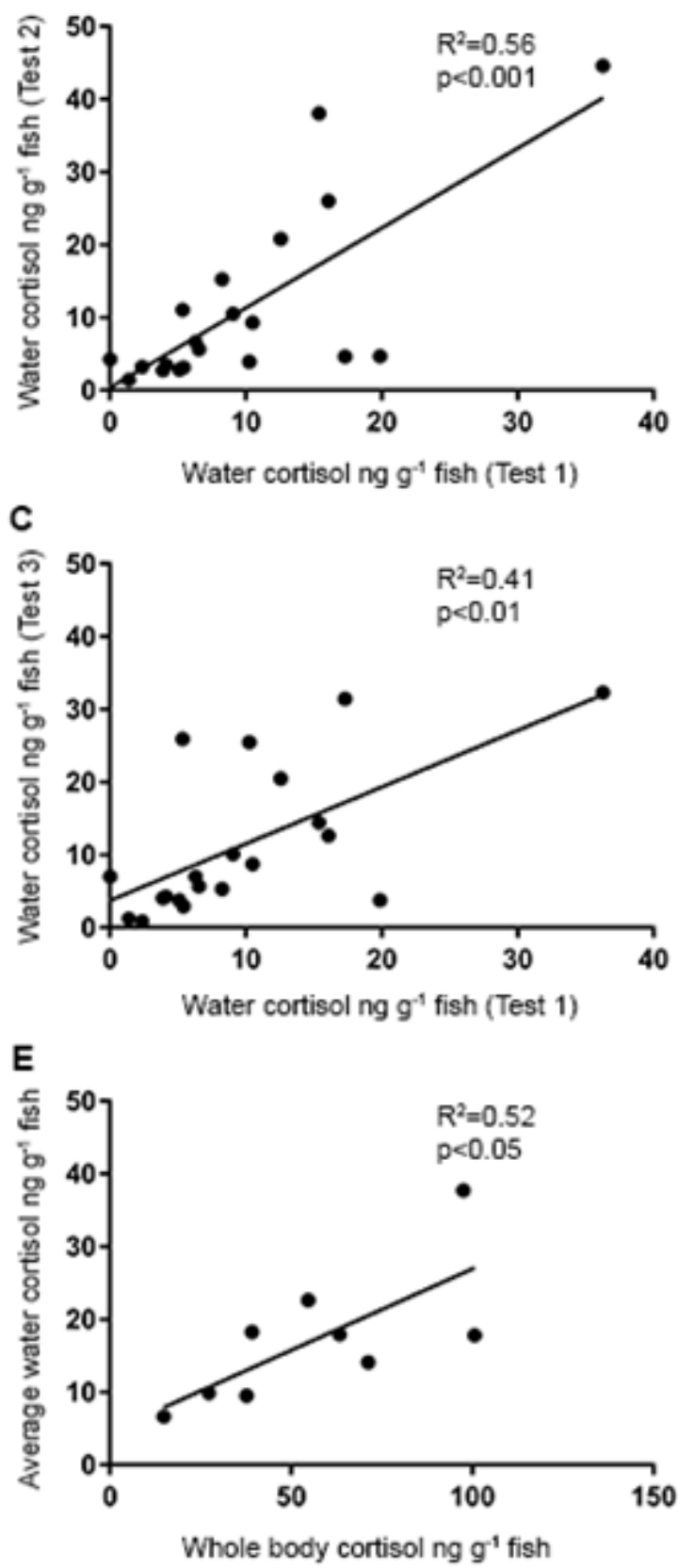

B

Females
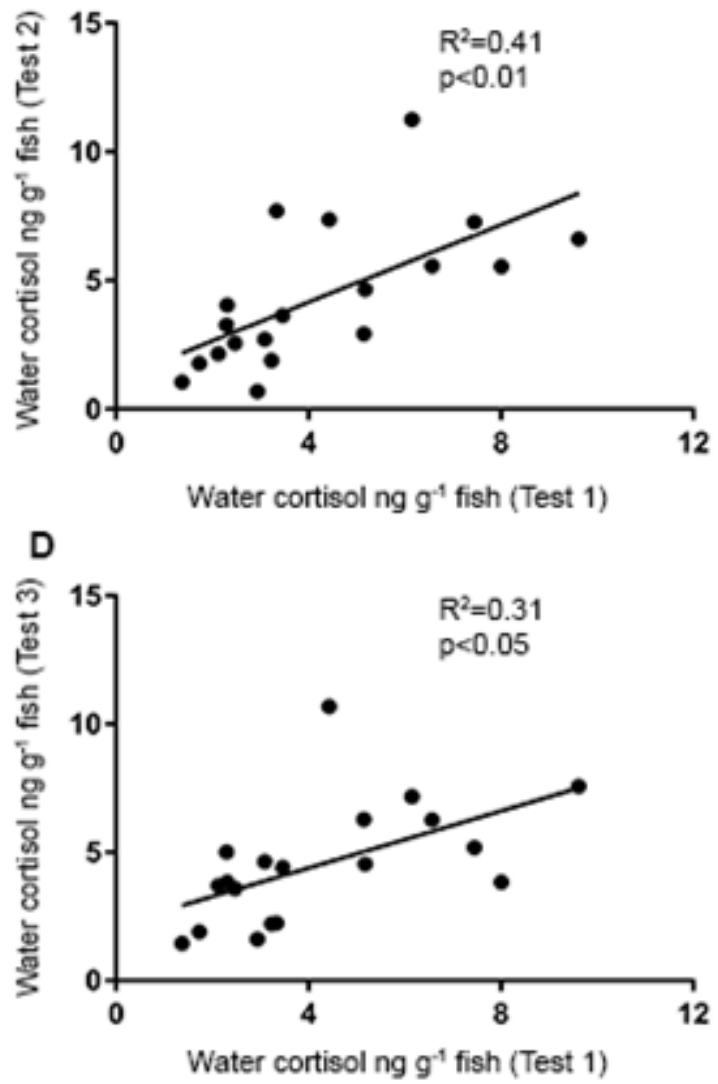

$\mathbf{F}$

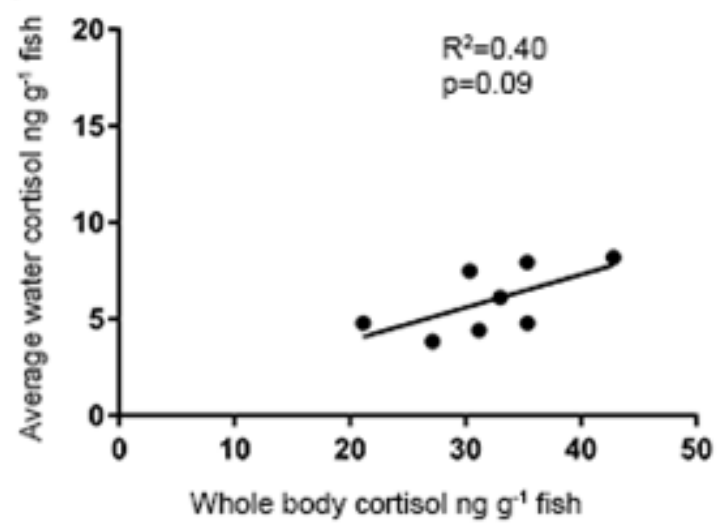

Figure 2

Cortisol-response to stress is variable between, but consistent within individual zebrafish (Danio rerio). Consistency and validity of post-stress water cortisol levels following acute stress was assessed by comparing water-borne cortisol levels following three consecutive stress tests (A-D) and mean water cortisol levels to whole body cortisol following a fourth stress test ( $E$ and $F$ ) in males ( $A, C$ and $E$ ) and females ( $B, D$ and $F)$. Consistency in cortisol responsiveness was tested by linear regression and 
correlation by a Pearson $r$ test in case of a normal distribution and by a Spearman rank test in case of a non-normal distribution.

A

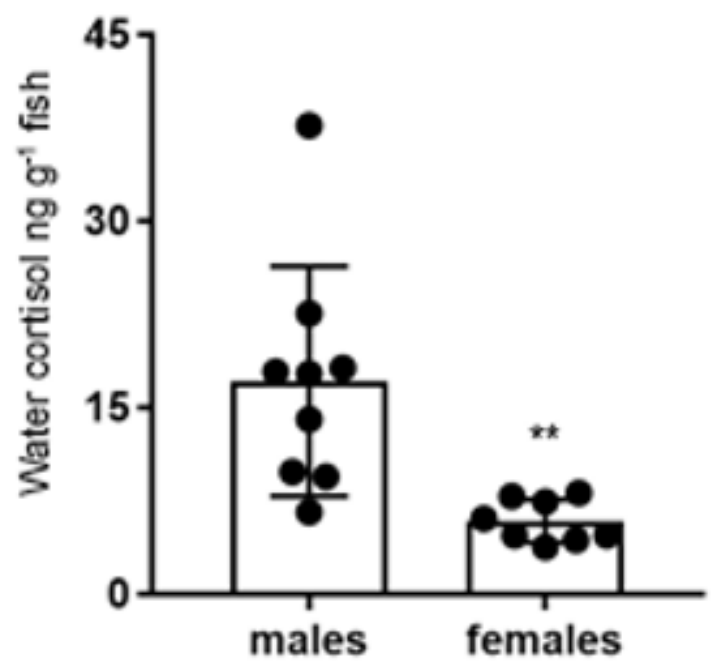

B

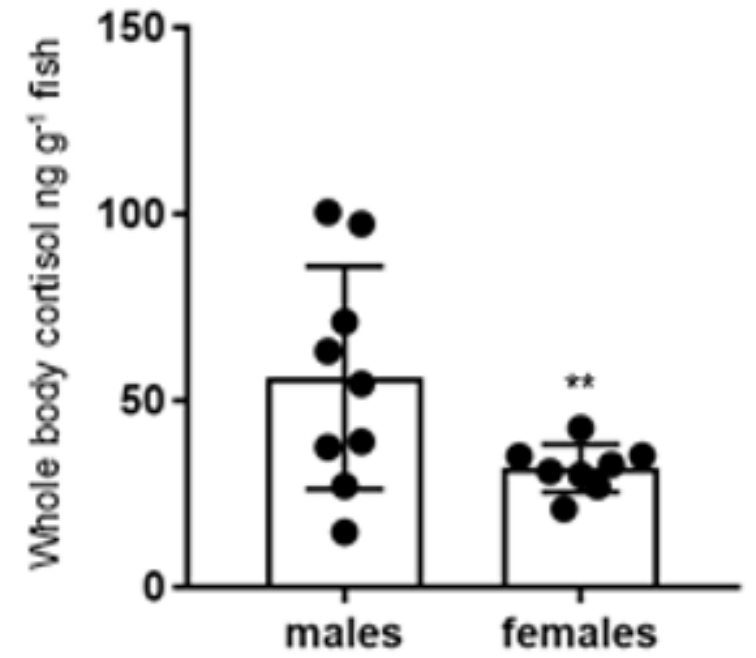

Figure 3

Cortisol levels is higher in males compared to female zebrafish. The magnitude in post-stress cortisol levels measured in (A) holding-water and (B) whole-body were lower for females compared to males. Statistical differences were tested by two-tailed un-paired t-tests. ${ }^{* \star} \mathrm{P}<0.01$ males versus females. 